\title{
ON NUMERICAL BOUNDS IN SCHOTTKY'S THEOREM*
}

\section{RAPHAEL M. ROBINSON}

According to Schottky's theorem, a function $f(z)$, which is regular and different from \pm 1 for $|z|<1$, and for which $f(0)=a$, is bounded in absolute value for $|z| \leqq r$ by a number depending only on $a$ and $r$. The asymptotic behaviour of the bound in Schottky's theorem for $a \rightarrow \infty$ has been studied by Ostrowski, $\dagger$ and numerical bounds have been given by Pfluger $\ddagger$ and Ahlfors; $\S$ but the numerical bounds which have been given are not asymptotically equal to the best bound.

Let $K(a, r)$ be the best bound possible in Schottky's theorem, in the form stated above. The purpose of this paper is to obtain, in as simple a manner as we can, estimates for $K(a, r)$ which are close enough to make it possible to derive from them the asymptotic formula

$$
8 K(a, r) \sim(8|a|)^{(1+r) /(1-r)}
$$

for $a \rightarrow \infty$ and fixed $r$. In fact we shall show that

$$
8 K(a, r)-10<(8|a|+10)^{(1+r) /(1-r)},
$$

while

$$
8 K(a, r)+10>(8|a|-10)^{(1+r) /(1-r)},
$$

provided $8|a|-10>0$; and from these inequalities the asymptotic for. mula evidently follows.

We assume as known that it is possible to map a triangle with zero angles, and sides orthogonal to the unit circle, conformally on the upper half-plane, the mapping being continuous on the boundary; the vertices of the triangle may be taken into $1,-1$, and $\infty$. (The mapping function is essentially an elliptic modular function.) The mapping function may be continued analytically throughout the unit circle by means of the Schwarz reflection principle, since this circle may be completely covered by triangles obtained from the original triangle by successive reflections on the sides. For later pur-

* Presented to the Society, April 15, 1939.

$\dagger$ Asymptotische Abschätzung des absolutes Betrages einer Funktion, die die Werte 0 und 1 nicht annimmt, Commentarii Mathematici Helvetici, vol. 5 (1933), pp. 55-87.

$\ddagger$ Über numerische Schranken im Schottky'schen Satz, Commentarii Mathematici Helvetici, vol. 7 (1935), pp. 159-170.

$\S A n$ extension of Schwarz's lemma, Transactions of this Society, vol. 43 (1938), pp. 359-364. 
poses, it is important to notice that if we start with a triangle containing the origin, a point of that triangle by successive reflections is always taken further from the origin, since it is always reflected from the exterior to the interior of a circle orthogonal to the unit circle.* Each triangle is mapped on an upper or lower half-plane, the entire circle $|z|<1$ being mapped on a Riemann surface with branch points of infinite order at $1,-1$, and $\infty$. The mapping function is regular for $|z|<1$, and different from \pm 1 . The inverse function is manyvalued, but has no singularities except at $1,-1$, and $\infty$.

By means of a linear transformation of the unit circle into itself, we can insure that the mapping function has any desired value, different from \pm 1 , at the origin. Let $T(z)=T(z, a)$ be the function thus obtained, for which $T(0)=a$ and $T^{\prime}(0)>0$. If we form the function $T(z, a)$ with $a=f(0)$, and let $T^{-1}(w)$ denote the inverse (many-valued) function, then the function $g(z)=T^{-1}(f(z))$ will be regular for $|z|<1$, since $f(z)$ is different from \pm 1 ; hence $g(z)$ will be single-valued, as soon as a definite branch of $T^{-1}(w)$ is chosen, which we shall do by imposing the condition $g(0)=0$. Then $g(z)$ satisfies the conditions of Schwarz's lemma, so that $|g(z)| \leqq|z|$. Since $f(z)=T(g(z))$, this leads to the conclusion that, for any function $f(z)$ with $f(0)=a$, which is regular and different from \pm 1 in the unit circle, we have

$$
|f(z)| \leqq \max _{\left|z^{\prime}\right| \leqq r} T\left(z^{\prime}, a\right) \quad \text { for } \quad|z| \leqq r .
$$

This is Schottky's theorem; and in fact the bound is exact, since $T(z, a)$ is an admissible function. The expression on the right is then equal to $K(a, r)$. In estimating this maximum, $z^{\prime}$ may be restricted to the intersection of the triangle (of our set of triangles covering the unit circle) containing the origin, and the circle $\left|z^{\prime}\right| \leqq r$. For the circle $\left|z^{\prime}\right| \leqq r$ is covered by the reflections of this part of the triangle containing the origin (since a point of the triangle outside of the circle is never reflected into the circle), and at two corresponding points, the values of $T\left(z^{\prime}, a\right)$ are equal or conjugate.

For the computation, it is convenient to map the unit circle $|z| \leqq 1$ on an upper half-plane $\Im \zeta \geqq 0$. Since the bound to be computed is the same for any value of $a$ as for its conjugate, we may suppose for convenience that $\Im a \geqq 0$, so that the triangle which contains the origin corresponds to an upper half-plane in the mapping $w=T(z, a)$. We may then suppose that this triangle goes into the triangle $-1 \leqq \Re \zeta \leqq 1$, $|\zeta| \geqq 1$, in such a way that the vertices $\zeta=1,-1, \infty$, correspond to $w=1,-1, \infty$, respectively. The function $T(z, a)$ then goes over into

\footnotetext{
* This fact is used in the last sentence of the following paragraph.
} 
a function $\Lambda(\zeta)$ which maps the triangle $-1 \leqq \Re \zeta \leqq 1,|\zeta| \geqq 1$, on the upper half-plane $\Im w \geqq 0$, in such a way that $1,-1$, and $\infty$, are fixed. Let $z=0$ go into the point $\zeta=b$; then $\Lambda(b)=T(0, a)=a$. The circle $|z| \leqq r$ goes over into a circle whose highest point is at a distance $\gamma \Im b$ from the real axis, where $\gamma$ is an abbreviation for $(1+r) /(1-r)$. The value of $K(a, r)$ is then less than or equal to the maximum of $|\Lambda(\zeta)|$ for $\zeta$ in the triangle and $\Im \zeta \leqq \gamma \Im b$, and this maximum is attained on the upper boundary $\Im \zeta=\gamma \Im b$, since $|\Lambda(\zeta)|$ increases upward on the vertical sides. On the other hand, the region over which we had to compute the maximum of $|\Lambda(\zeta)|$ contains a point of $\Im \zeta=\gamma \Im b$. Hence $K(a, r)$ is included between the minimum and maximum of $|\Lambda(\zeta)|$ for $\zeta$ in the triangle and $\Im \zeta=\gamma \Im b$. (That the problem can be reduced to this has been recognized by others; but they seem to have overlooked the simple method of calculating a bound given below.)

Let $\phi(\zeta)$ be the function which maps our triangle in the $\zeta$-plane onto the region $\Im w \geqq 0,|w| \geqq 1$, in such a way that $1,-1$, and $\infty$ are fixed. Then $\Lambda(\zeta)=[\phi(\zeta)+1 / \phi(\zeta)] / 2$. We note further that $\Lambda(\zeta)$ maps the arc of the circle $|\zeta+1|=2$ extending from 1 to $-1+2 i$ (which may be characterized as a circular arc through the vertex 1 which is orthogonal to the opposite side of the triangle as well as to the real axis) onto an arc of the same circle extending from 1 to -3 . For if we transform the mapping by taking the upper half-plane into the unit circle by a linear transformation, in such a way that the vertices of the triangle go into points which are equally spaced around the unit circle, the corresponding statement will be evident from the symmetry of the figure. From this we conclude that $\Lambda(\zeta)$ maps the region $-1 \leqq \Re \zeta \leqq 1,|\zeta+1| \geqq 2$, onto the region $\Im w \geqq 0,|w+1| \geqq 2$, the points 1 and $\infty$ being fixed, and $-1+2 i$ going into -3 . That is the same as saying that $\Lambda(2 \zeta-1)$ takes the region $0 \leqq \Re \zeta \leqq 1,|\zeta| \geqq 1$, into the same region in the $w$-plane, the points 1 and $\infty$ being fixed, and $i$ going into -3 . But $2 \phi(\zeta)^{2}-1$ does exactly the same, hence $\Lambda(2 \zeta-1)$ $=2 \phi(\zeta)^{2}-1$. Thus both $\Lambda(\zeta)$ and $\Lambda(2 \zeta-1)$ are expressed in terms of $\phi(\zeta)$, and hence brought into relation to each other.

By means of the function $t=i e^{\pi \zeta / 2 i}$ the half-strip $-1 \leqq \Re \zeta \leqq 1, \Im \zeta \geqq 0$ is taken into the region $\Im t \geqq 0,|t| \geqq 1$. This transformation takes our triangle in the $\zeta$-plane into a region in the upper half of the $t$-plane, bounded by the part of the real axis to the left of -1 and the part to the right of 1 , and by a curve $C$ joining -1 and 1 , whose distance from the origin is always between 1 and $e^{\pi / 2}$. We may regard $\Lambda(\zeta)$ as a function of $t$, for $t$ in the part of the upper half-plane outside of $C$, and continue across the real axis by the Schwarz reflection principle, so that $\Lambda(\zeta)$ is a regular function of $t$, for $t$ outside of $C$ and its reflec- 
tion in the real axis, except for a simple pole at $\infty .{ }^{*}$ It is clear from the symmetry that the expansion of $\Lambda(\zeta)$ in the neighborhood of $t=\infty$ is of the form $\Lambda(\zeta)=c t+c_{1} / t+c_{3} / t^{3}+\cdots$, all the coefficients being real, and the first coefficient $c$ being positive. From the functional relation obtained above, the coefficients $c, c_{1}, c_{3}, \cdots$ could be calculated. However, we need only the value of $c$. Now if we replace $\zeta$ by $2 \zeta-1$, then $t$ is replaced by $t^{2}$, so that besides the relation $\Lambda(\zeta) \sim c t$, we have also $\Lambda(2 \zeta-1) \sim c t^{2}$. But from the equations expressing $\Lambda(\zeta)$ and $\Lambda(2 \zeta-1)$ in terms of $\phi(\zeta)$, we have $\Lambda(\zeta) \sim \phi(\zeta) / 2$ and $\Lambda(2 \zeta-1)$ $\sim 2 \phi(\zeta)^{2}$. Eliminating the $\phi(\zeta)$ leads to the conclusion that $c t^{2} \sim 8 c^{2} t^{2}$, from which we find $c=1 / 8$. From this we see that $8 \Lambda(\zeta)-t \rightarrow 0$ for $t \rightarrow \infty$. Thus the maximum of $|8 \Lambda(\zeta)-t|$ will occur for $t$ on the curve $C$. Since the curve is symmetric with respect to the imaginary axis, the part in the first quadrant is taken into the segment from 0 to 8 by the function $8 \Lambda(\zeta)$. It is clear then that $|8 \Lambda(\zeta)-t| \leqq\left(64+e^{\pi}\right)^{1 / 2}<10$ for $t$ on $C$ and hence for all values of $t$ considered. Now $|t|=e^{(\pi / 2) \Im\}}$, so that

$$
8|\Lambda(\zeta)|-10<e^{(\pi / 2) \Im \zeta}<8|\Lambda(\zeta)|+10
$$

for all points $\zeta$ of our triangle. Hence for $\Im \zeta=\gamma \Im b$, we have

$$
8|\Lambda(\zeta)|-10<e^{(\pi / 2) \Im_{\zeta}}=\left[e^{(\pi / 2) \Im_{b}}\right]^{\gamma}<[8|\Lambda(b)|+10]^{\gamma},
$$

and, provided $8|\Lambda(b)|-10>0$,

$$
8|\Lambda(\zeta)|+10>e^{(\pi / 2) \Im_{\zeta}}=\left[e^{(\pi / 2) \Im_{b}}\right]^{\gamma}>[8|\Lambda(b)|-10]^{\gamma} .
$$

Remembering that $\Lambda(b)=a$, and that $K(a, r)$ is between the maximum and minimum of $|\Lambda(\zeta)|$ for $\Im \zeta=\gamma \Im b$, we see that these inequalities yield the desired bounds for $K(a, r)$.

University of CaLIFornia

* The function $\Lambda(\zeta)$ is evidently a single-valued function of $t$ in the region mentioned, since in continuing across either segment of the real axis we obtain its value at a point $t$ of the lower half-plane as the conjugate of its value at $\bar{t}$. 